\title{
Screening of gene markers related to the prognosis of metastatic skin cutaneous melanoma based on Logit regression and survival analysis
}

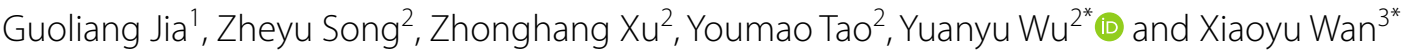

\begin{abstract}
Background: Bioinformatics was used to analyze the skin cutaneous melanoma (SKCM) gene expression profile to provide a theoretical basis for further studying the mechanism underlying metastatic SKCM and the clinical prognosis.

Methods: We downloaded the gene expression profiles of 358 metastatic and 102 primary (nonmetastatic) CM samples from The Cancer Genome Atlas (TCGA) database as a training dataset and the GSE65904 dataset from the National Center for Biotechnology Information database as a validation dataset. Differentially expressed genes (DEGs) were screened using the limma package of R3.4.1, and prognosis-related feature DEGs were screened using Logit regression (LR) and survival analyses. We also used the STRING online database, Cytoscape software, and Database for Annotation, Visualization and Integrated Discovery software for protein-protein interaction network, Gene Ontology, and Kyoto Encyclopedia of Genes and Genomes (KEGG) pathway analyses based on the screened DEGs.

Results: Of the 876 DEGs selected, 11 (ZNF750, NLRP6, TGM3, KRTDAP, CAMSAP3, KRT6C, CALML5, SPRR2E, CD3G, RTP5, and FAM83C) were screened using LR analysis. The survival prognosis of nonmetastatic group was better compared to the metastatic group between the TCGA training and validation datasets. The 11 DEGs were involved in 9 KEGG signaling pathways, and of these 11 DEGs, CALML5 was a feature DEG involved in the melanogenesis pathway, 12 targets of which were collected.
\end{abstract}

Conclusion: The feature DEGs screened, such as CALML5, are related to the prognosis of metastatic CM according to LR. Our results provide new ideas for exploring the molecular mechanism underlying CM metastasis and finding new diagnostic prognostic markers.

Keywords: Cutaneous melanoma, Metastasis, Prognosis, Bioinformatics, Differentially expressed genes

*Correspondence: wyy511@jlu.edu.cn; wanxy17@mails.jlu.edu.cn ${ }^{2}$ Department of Gastrointestinal and Colorectal Surgery, The Third Hospital of Jilin University, No.126, Xiantai Street, Changchun 130033, Jilin, China

${ }^{3}$ Department of Brest Surgery, The Second Clinical Hospital of Jilin University, NO.218, Ziqiang Street, Nanguan District, Changchun 130000 Jilin, China

Full list of author information is available at the end of the article

\section{Background}

Skin cutaneous melanoma (SKCM) is a common skin malignancy with poor prognosis due to aggressiveness and metastasis [1]. In recent years, the incidence of SKCM has significantly increased, and the survival rate of patients remains poor; the 5-year survival rate of metastatic SKCM patients is less than 5\% [2]. As metastasis is an important cause of poor prognosis of SKCM, it is 
necessary to identify its underlying molecular mechanisms and determin its molecular biomarkers.

Previous studies have focused on molecular markers related to SKCM metastasis and prognosis. For example, S100 has been clearly identified as a diagnostic marker of SKCM, and in terms of SKCM prognosis, the most studied diagnostic marker is S100B. Other studies have reported that high $\mathrm{S} 100 \mathrm{~B}$ expression indicates tumor recurrence and metastasis [3, 4]. Da Forno et al. [5] showed that high expression of Wnt-5a indicates an increase in SKCM aggressiveness, distant metastases, and poor prognosis. Melanoma inhibitory protein is also considered a diagnostic marker of SKCM metastasis and poor prognosis [6]. Ci et al. [7] showed that CDCA8 overexpression promotes the malignant progression of SKCM and leads to poor prognosis. Yang et al. [8] found that STK26, KCNT2, and CASP12 expression is correlated with the prognosis of SKCM using weighted gene co-expression network analysis. However, the mechanism underlying metastasis of a nevus into SKCM is still unclear, and further research is urgently needed.

Logit regression (LR) is popular among medical practitioners because of its interpretability and ease of application without the need for a computer [9]. Zejnullahu et al. [10] examined the prevalence and risk factors of postpartum depression using LR. Sufriyana et al. [11] used LR to explore the predictive performances for pregnancy care to inform clinicians' decision making. Sokou et al.[12] developed and validated a prediction model for clinical variables. These reports suggested that LR model for predicting the risk assessment is regularly used in medicine to guide management decisions.

In this study, we downloaded gene expression detection data of 460 (358 metastatic and 102 primary [nonmetastatic]) SKCM patients from The Cancer Genome
Atlas (TCGA) database. The limma package of R3.4.1 was used to screen for differentially expressed genes (DEGs), the survival package of R3.4.1 was used for single- and multifactor Cox regression analysis, and feature DEGs were selected using LR. Next, we constructed a protein-protein interaction (PPI) network, followed by Gene Ontology (GO) and Kyoto Encyclopedia of Genes and Genomes (KEGG) pathway enrichment analyses.

\section{Methods}

\section{Gene expression profile data and clinical information}

We downloaded 472 SKCM patients' gene expression profile data using Illumina HiSeq 2000 from the TCGA database (https://gdc-portal.nci.nih.gov/). According to the clinical information, 460 tumor samples had information about metastasis, of which 358 metastatic and 102 nonmetastatic SKCM tumor samples were used as a training dataset. Table 1 showed all the clinical information present in the dataset; there was significant association in pathologic $\mathrm{N}$ status, tumor recurrence, and death between metastatic SKCM and nonmetastatic SKCM. In addition, we downloaded another data set of SKCM patient gene expression profiles (GSE65904) from the National Center for Biotechnology Information database (NCBI) (https://www.ncbi.nlm.nih.gov/), which contained 214 SKCM tumor samples. The detection platform used was the Illumina HumanHT-12 V4.0 expression beadchip. According to the clinical information, 150 tumor samples had information about metastasis, of which 83 metastatic and 67 nonmetastatic SKCM tumor samples were used as a validation dataset.

\section{Data preprocessing and DEG screening}

Tumor samples in the training dataset were divided into metastatic and nonmetastatic groups according to the

Table 1 Statistical comparison of clinical information of two types of CM samples in TCGA training data set

\begin{tabular}{|c|c|c|c|}
\hline Clinical characteristics & $\begin{array}{l}\text { With metastatic } \\
(\mathrm{N}=358)\end{array}$ & $\begin{array}{l}\text { Without metastatic } \\
(\mathrm{N}=102)\end{array}$ & $P$ value \\
\hline Age (years, mean \pm sd) & $56.26 \pm 15.73$ & $64.53 \pm 13.91$ & $7.10 \mathrm{E}-07^{\mathrm{a}}$ \\
\hline Gender (Male/Female) & $226 / 132$ & $60 / 42$ & $0.488^{b}$ \\
\hline Pathologic_M (M0/M1/-) & $313 / 21 / 24$ & $97 / 3 / 2$ & $0.317^{b}$ \\
\hline Pathologic_N (N0/N1/N2/N3/-) & $172 / 65 / 39 / 44 / 38$ & $57 / 8 / 10 / 11 / 16$ & $0.0785^{b}$ \\
\hline Pathologic_T (T0/T1/T2/T3/T4/-) & 23/40/72/80/67/76 & $0 / 1 / 5 / 10 / 84 / 2$ & $2.2 \mathrm{E}-16^{\mathrm{b}}$ \\
\hline Pathologic_stage (I/II/III/IV/-) & $81 / 74 / 142 / 20 / 41$ & $2 / 65 / 27 / 3 / 5$ & $1.133 \mathrm{E}-15^{\mathrm{b}}$ \\
\hline Radiotherapy (Yes/No/-) & $47 / 310 / 1$ & $2 / 100 / 0$ & $4.395 E-04^{b}$ \\
\hline Tumor recurrence (Yes/No/-) & $69 / 135 / 154$ & $20 / 72 / 10$ & $0.0402^{b}$ \\
\hline Dead (Death/Alive) & 193/165 & $29 / 73$ & $0.0715^{b}$ \\
\hline Overall survival time (months, mean \pm sd) & $74.10 \pm 67.91$ & $47.15 \pm 10.04$ & $5.279 E-22^{a}$ \\
\hline
\end{tabular}

\footnotetext{
${ }^{a}$ T test between groups
}

${ }^{\mathrm{b}}$ Fisher exact test 
clinical information. DEGs in both groups were analyzed using the limma package (https://bioconductor.org/packa ges/release/bioc/html/limma.html) version 3.34 .7 of R3.4.1. FDR $<0.05$ and $\left|\log _{2} \mathrm{FC}\right|>1$ were used as the cutoff threshold.

Next, we performed two-way hierarchical clustering based on the centered Pearson correlation algorithm based on the DEG expression in the training dataset using pheatmap version 1.0.8 (https://cran.r-project.org/ web/packages/pheatmap/index.html) of R3.4.1, and then performed the following analysis:

(1) To determine whether the clinical information about the tumor samples in different clusters was significantly different, we ran the chisq.test function (http://www.bioconductor.org/help/search/index. html?q=chisq.test/) of R3.4.1 on the clinical information.

(2) According to the survival prognosis information about tumor samples in different clusters, we used the Kaplan-Meier (KM) curve in the survival package version 2.41-1 (http://bioconductor.org/packa ges/survivalr/) of R3.4.1 to calculate the correlation between clusters and survival prognosis.

\section{Screening of independent prognostic DEGs}

On the basis of the clinical prognosis information about the SKCM tumor samples included in this analysis, we univariate Cox regression analysis in the survival package version 2.41-1 of R3.4.1 [13] to screen DEGs with significant differences in prognosis. We further used multivariate Cox regression analysis to screen independent prognosis-related DEGs, and log-rank $P$ value $<0.05$ was selected as the threshold for significant correlation.

\section{Screening of feature DEGs}

On the basis of previously screened DEGs independently associated with survival prognosis, we used the glm function of R3.4.1 to perform LR [14, 15] to screen out feature DEGs and classify metastatic and nonmetastatic SKCM tumor samples. All genes with $P<0.05$ were considered feature DEGs. Next, on the basis of the expression of the feature DEGs, we calculated the discriminant accuracy and classified all tumor samples in the training and validation datasets into metastatic and nonmetastatic groups. The KM survival curve in the survival package version 2.41-1 of R3.4.1 was used to perform correlation analysis of the actual survival prognosis of the metastatic and nonmetastatic SKCM tumor samples obtained from the LR classification.

\section{Cluster analysis of feature DEGs}

For the training and validation datasets, we used pheatmap version 1.0.8 [16] of R3.4.1 to perform two-way hierarchical clustering based on the centered Pearson correlation algorithm based on feature DEG expression. On the basis of the survival prognosis information about samples in different clusters, we used the KM survival curve in the survival package version 2.41-1 (http://bioco nductor.org/packages/survivalr/) of R3.4.1 to determine the correlation between clusters and survival prognosis in the training and validation datasets. The expression levels of feature DEGs in different clusters were then demonstrated.

\section{PPI network construction function analysis}

We constructed a PPI network to evaluate the interactions between proteins encoded by the DEGs. The PPI network was based on the STRING database version 10.0 (http://string-db.org/) [17] and was visualized using Cytoscape software version 3.6.1 (http://www.cytoscape. org/) [18]. Next, we used the Database for Annotation, Visualization and Integrated Discovery (DAVID) version 6.8 (https://david.ncifcrf.gov/) to conduct GO function annotation, including Biology Process, and KEGG signaling pathway enrichment analysis [19, 20]. Finally, significantly relevant $\mathrm{GO}$ functions were screened using Fisher's exact test with $P<0.05$.

\section{Results \\ DEG screening}

On the basis of the clinical information, SKCM tumor samples in the TCGA training dataset were divided into metastatic $(n=358)$ and nonmetastatic $(n=102)$ groups. We screened 876 DEGs, including 353 significantly downregulated and 523 significantly upregulated DEGs. Figure 1a showed the test volcano map. The two-dimensional hierarchical clustering heat map (Fig. 1b) showed that samples with similar gene expression patterns were hierarchically clustered into the same group, indicating that the screened DEGs can well distinguish tumor samples with different prognoses. The two clusters of the clustering chart contained 247 and 213 tumor samples, respectively. Comparing the clinical information of the tumor samples in the two clusters showed that the samples were significantly different in age, pathologic $\mathrm{T}$ status, pathologic stage, radiotherapy, tumor metastasis, and survival time (Table 2). In addition, comparing the prognostic levels of the tumor samples showed that the survival ratio of cluster 1 was significantly better than cluster $2(P=9.141 \mathrm{e}-09)$ (Fig. 1c). 


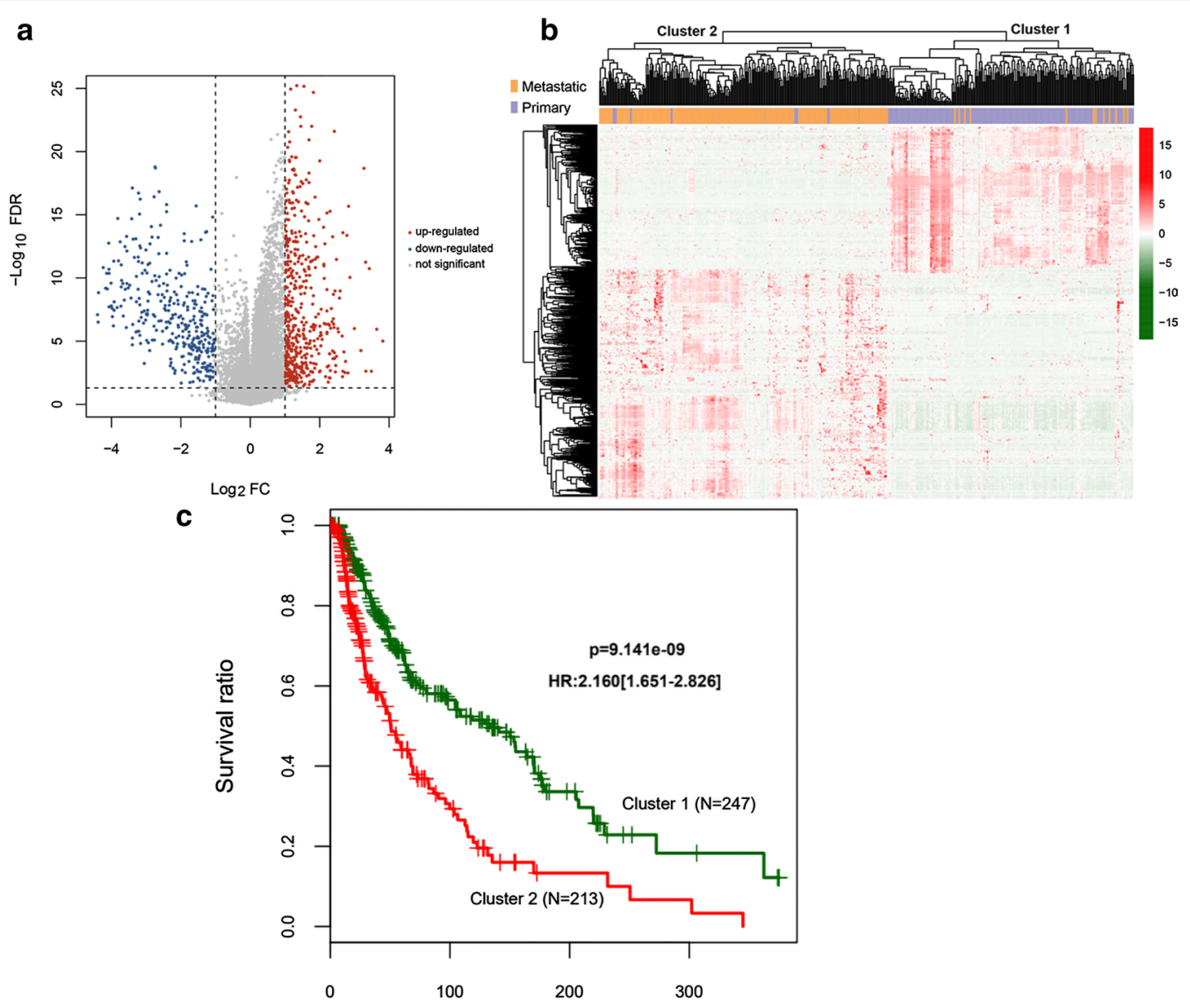

Fig. 1 DEGs screening. a Volcano map of DEGs. The blue and red dots indicate DEGs that are significantly downregulated and upregulated, respectively. The black horizontal line indicates FDR $<0.05$, and the two black vertical lines indicate $|\log 2 \mathrm{FC}|>1$. $\mathbf{b}$ Two-way hierarchical clustering heat map based on DEG expression. In the sample bar, orange and purple represent metastatic and nonmetastatic SKCM tumor samples, respectively. c KM curves of sample prognosis information about cluster 1 and cluster 2, which is obtained by clustering based on DEG expression in the TCGA training dataset. The green and red curves represent cluster 1 and cluster 2 tumor sample groups, respectively. DEGs, differentially expressed genes; SKCM, skin cutaneous melanoma; KM, Kaplan-Meier; TCGA, The Cancer Genome Atlas

Table 2 Statistical comparison of clinical information among samples in clusters based on DEGs expression levels

\begin{tabular}{|c|c|c|c|}
\hline Clinical characteristics & Cluster $1(\mathrm{~N}=247)$ & Cluster $2(\mathrm{~N}=213)$ & $P$ value \\
\hline Age (years, mean $\pm s d$ ) & $54.96 \pm 15.41$ & $61.74 \pm 15.29$ & $3.07 \mathrm{E}-06^{\mathrm{a}}$ \\
\hline Gender (Male/Female) & $152 / 95$ & $134 / 79$ & $0.773^{b}$ \\
\hline Pathologic_M (M0/M1/-) & 219/14/14 & 191/10/12 & $0.679^{b}$ \\
\hline Pathologic_N (N0/N1/N2/N3/-) & $118 / 42 / 28 / 33 / 26$ & $111 / 31 / 21 / 22 / 28$ & $0.599^{b}$ \\
\hline Pathologic_T (T0/T1/T2/T3/T4/-) & 20/33/51/54/44/45 & $3 / 8 / 26 / 36 / 107 / 33$ & $2.893 \mathrm{E}-13^{\mathrm{b}}$ \\
\hline Pathologic_stage (I/II/III/IV/-) & $66 / 43 / 97 / 14 / 27$ & $17 / 96 / 72 / 9 / 19$ & $6.568 \mathrm{E}-12^{\mathrm{b}}$ \\
\hline Radiotherapy (Yes/No/-) & $35 / 212 / 0$ & 14/198/1 & $0.00975^{b}$ \\
\hline Tumor recurrence (Yes/No/-) & $45 / 107 / 95$ & $44 / 100 / 69$ & $0.899^{b}$ \\
\hline Tumor metastatic (Yes/No) & $235 / 12$ & $123 / 90$ & $2.200 \mathrm{E}-16^{\mathrm{b}}$ \\
\hline Dead (Death/Alive) & $111 / 136$ & $111 / 102$ & $0.149^{b}$ \\
\hline Overall survival time (months, mean \pm sd) & $78.21 \pm 71.12$ & $42.07 \pm 49.53$ & $4.269 \mathrm{E}-10^{\mathrm{a}}$ \\
\hline
\end{tabular}

\footnotetext{
${ }^{a} T$ test between groups

${ }^{\mathrm{b}}$ Fisher exact test
} 
Table 3 List of important prognostic feature DEGs screened by Logit model

\begin{tabular}{lllll}
\hline Gene & B & SE & Df & $P$ value \\
\hline ZNF750 & -7.07556 & 3.11741 & 1 & 0.02323 \\
NLRP6 & -3.81707 & 1.92378 & 1 & 0.04724 \\
TGM3 & -3.63216 & 1.08106 & 1 & 0.00078 \\
KRTDAP & -1.36009 & 0.65739 & 1 & 0.03855 \\
CAMSAP3 & -1.18449 & 0.52553 & 1 & 0.0242 \\
KRT6C & -0.96555 & 0.44870 & 1 & 0.03141 \\
CALML5 & 1.47087 & 0.57774 & 1 & 0.0109 \\
SPRR2E & 1.59208 & 0.53097 & 1 & 0.00271 \\
CD3G & 4.12233 & 1.77857 & 1 & 0.02046 \\
RTP5 & 7.35929 & 2.91801 & 1 & 0.01167 \\
FAM83C & 9.35699 & 3.95983 & 1 & 0.01813 \\
\hline
\end{tabular}

$\mathrm{B}$, regression coefficient; $\mathrm{SE}$, standard error; $\mathrm{Df}$, degree of freedom

Table 4 Fuzzy matrix of LR classification result

\begin{tabular}{lllll}
\hline Dataset name & Predict & & \\
\cline { 2 - 5 } & Class & Metastatic & Primary & Percent \\
\hline TCGA dataset & Observed & & & \\
& Metastatic & 350 & 7 & 97.77 \\
& Primary & 25 & 77 & 75.49 \\
Overall percent & & & & 92.83 \\
GSE65904 micro- & Observed & & & \\
array dataset & & & 11 & 86.75 \\
& Metastatic & 72 & 40 & 59.70 \\
& Primary & 27 & & 74.67 \\
Overall percent & & &
\end{tabular}

\section{Screening of independent prognostic DEGs}

On the basis of the clinical prognosis information about the 460 SKCM tumor samples, we analyzed the 876 screened DEGs by univariate single-factor Cox regression analysis using the survival package version 2.41-1 of R3.4.1 and screened 435 DEGs significantly associated with prognosis. These 435 DEGs were further analyzed using multivariate Cox regression analysis, and 61 independent prognostic DEGs were screened.

\section{Screening of feature DEGs by LR}

The 61 independent prognostic DEGs were further screened using LR to obtain 11 feature DEGs: ZNF750, NLRP6, transglutaminase 3 (TGM3), KRTDAP, CAMSAP3, KRT6C, CALML5, SPRR2E, CD3G, RTP5, and FAM83C (Table 3). LR was also used to classify the tumor samples in the training and validation datasets on the basis of the expression levels of these 11 feature DEGs (Table 4). Metastatic SKCM accounted for 98\% of the tumor samples in the training dataset and $87 \%$ of the tumor samples in the validation dataset. In addition, KM curve analysis was performed on the basis of LR classification prediction results (Fig. 2a, b). In the TCGA training and GSE65904 validation datasets, the predicted survival prognosis of the nonmetastatic group was better than the metastatic group $(P=1.578 \mathrm{e}-08$ and $2.786 \mathrm{e}-08$, respectively).

\section{Cluster analysis of feature DEGs}

In the TCGA training and GSE65904 validation datasets, we performed two-way hierarchical clustering based on

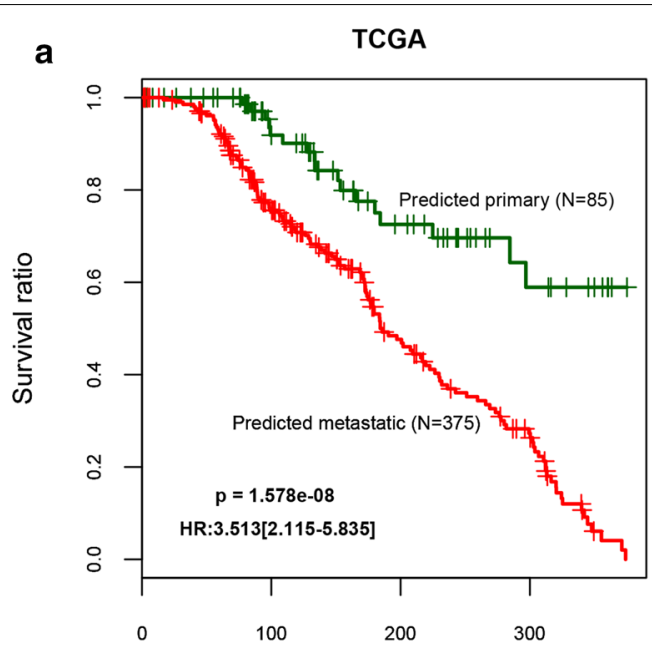

Overall survival time(months)

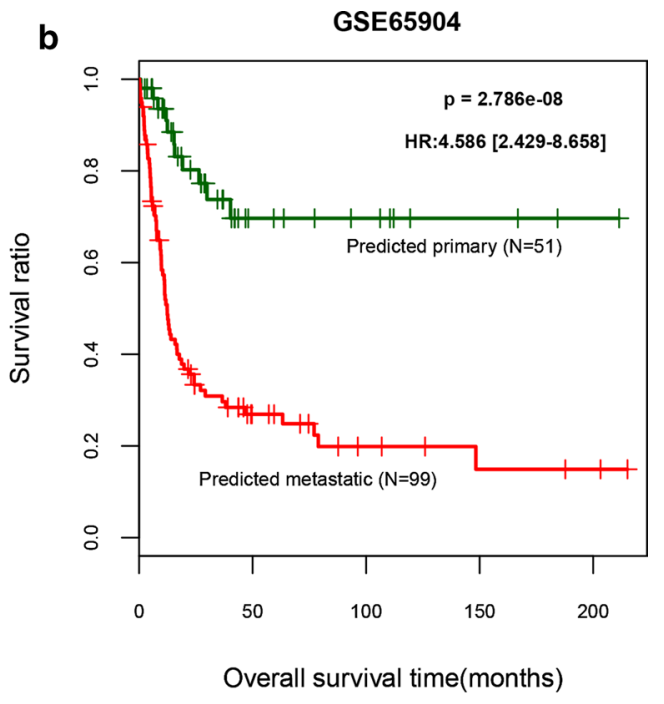

Fig. 2 KM curves of survival prognosis based on LR classification results for the TCGA training dataset (a) and the GSE65904 validation dataset (b). The green and red curves represent the predicted SKCM tumor samples of nonmetastatic and metastatic types, respectively. LR, Logit regression; SKCM, skin cutaneous melanoma; KM, Kaplan-Meier; TCGA, The Cancer Genome Atlas 

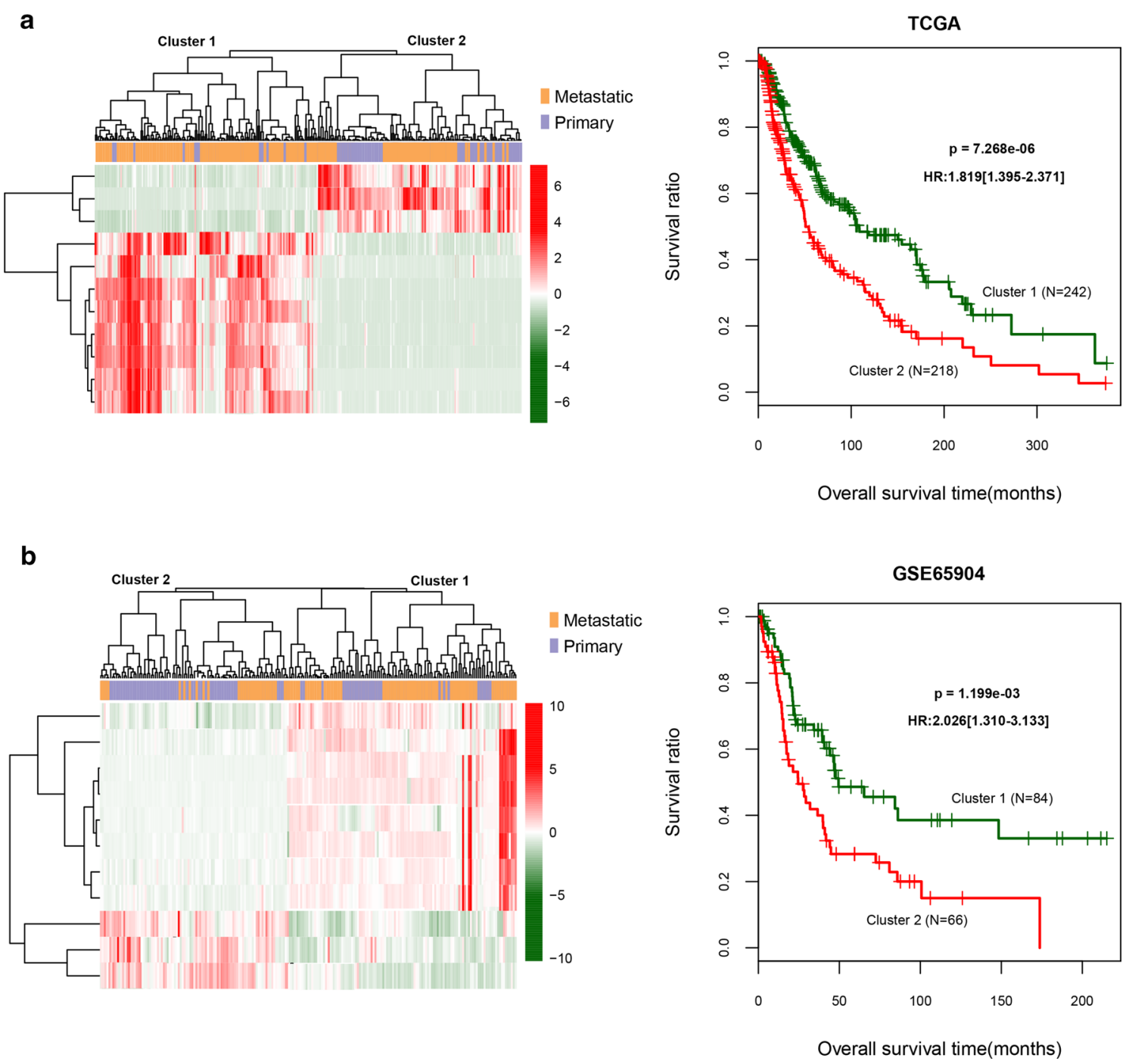

Fig. 3 TCGA training dataset (a) and GSE65904 validation dataset (b). Left: Two-way hierarchical clustering chart based on the expression of 11 feature DEGs. Right: KM curve of sample prognosis information about cluster 1 and cluster 2 based on left clustering. The green and red curves represent the cluster 1 and cluster 2 tumor sample groups, respectively. DEGs, differentially expressed genes; KM, Kaplan-Meier; TCGA, The Cancer Genome Atlas

the centered Pearson correlation algorithm based on the expression of the 11 feature DEGs (Fig. 3a). We also performed KM curve analysis on the tumor samples in clusters 1 and 2 on the basis of the expression of the 11 DEGs. In TCGA the training and GSE65904 validation datasets, the survival prognosis in cluster 1 was better compared to cluster $2(P=7.268 \mathrm{e}-06$ and $1.199 \mathrm{e}-03$, respectively) (Fig. 3b). The expression levels of the $11 \mathrm{fea}-$ ture DEGs in the two clusters of the TCGA training and GSE65904 validation datasets are shown in Fig. 4. Tumor samples with similar gene expression patterns were hierarchically clustered into the same group, indicating that the selected DEGs can well distinguish tumor samples with different prognoses. We identified eight genes (CALML5, CAMSAP3, FAM83C, KRTDAP, SPRR2E, $T G M 3$, and ZNF750) with high expression associated with poor prognosis and three genes (CD3G, NLRP6, and RTP5) with low expression associated with poor prognosis (Fig. 4a). In the GSE65904 validation dataset, we obtained similar results: three genes (CD3G, NLRP6, and RTP5) with low expression were associated with poor prognosis (Fig. 4b).

\section{PPI analysis}

We constructed a PPI network based on the 876 screened DEGs using the STRING online database. 


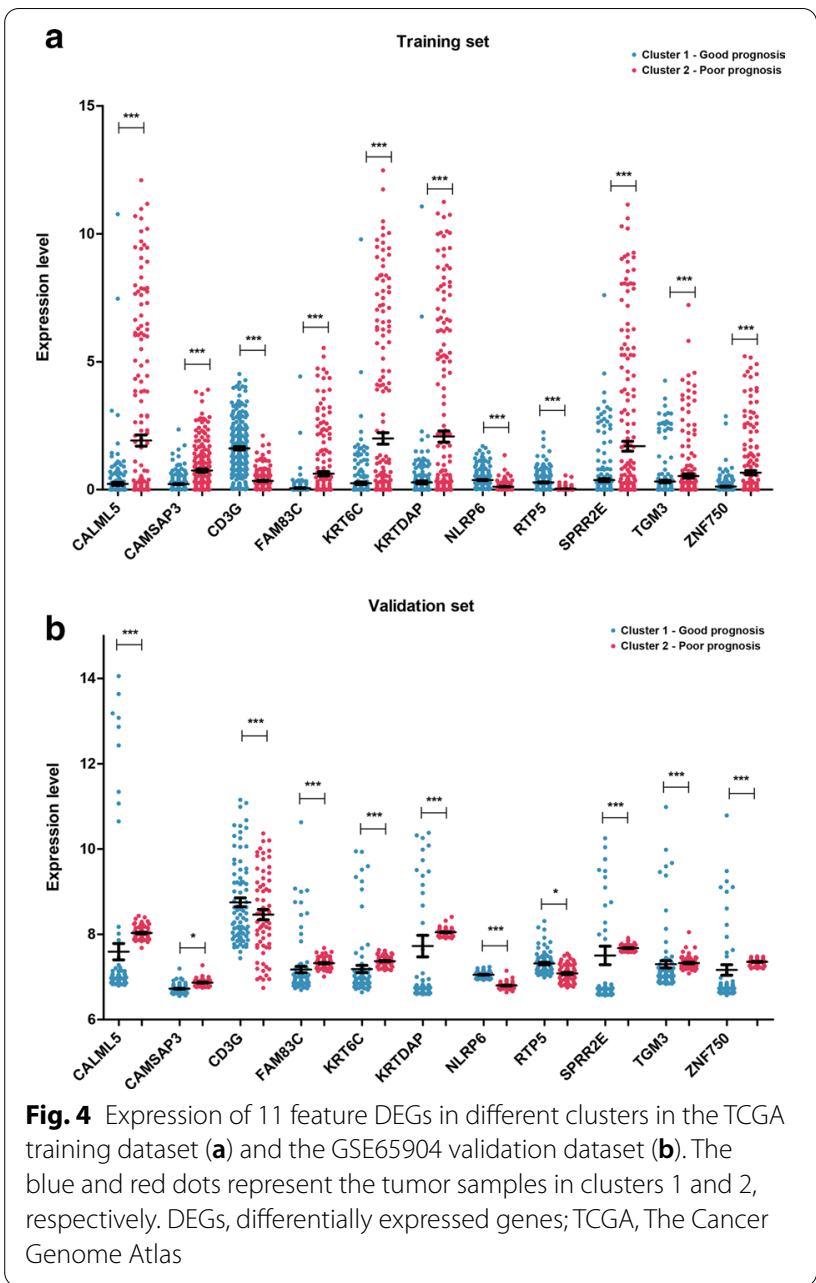

After screening the 11 feature DEGs using LR, we only focused on their PPI network, which contained 107 pairs of interaction connections related to the 11 feature DEGs. Cytoscape software was used to construct the PPI network (Fig. 5) containing 78 nodes (65 downregulated and 13 upregulated) and 107 edges. We collected 12 targets of CALML5, including TGM3, PDE1C, CASP14, and CASP14, and 26 targets of SPRR2E, including SPRR2A, $I V L$, and $L C E 2 A$.

\section{GO and KEGG signaling pathway enrichment analysis}

We obtained 21 significantly related GO biological processes and 9 KEGG signaling pathways using the online tool DAVID (Table 5). For GO functions, the DEGs were primarily enriched in "epidermis development," "keratinization," "keratinocyte differentiation," and "establishment of skin barrier," which involved many genes, such as CALML5, SPRR2A, and HRNR. For KEGG signaling pathways, the DEGs were primarily enriched in "calcium signal pathway," "GnRH signal pathway," and "melanin production pathway." Of the 9 KEGG signaling pathways, in the "hsa04916: Melanogenesis" pathway, CALML3 and CALML5 deserved attention, of which CALML5 was one of important feature DEGs.

\section{Discussion}

SKCM is the most malignant skin tumor type derived from melanocytes, with high mortality, high metastasis, and difficulty in treating. As SKCM grows, cancer cells penetrate the skin and mucous membranes, eventually reach blood vessels or lymphatic channels, and quickly spread throughout the body and major organs [21]. Metastasis is an important cause of poor prognosis in SKCM patients [22]. The 5-year survival rate of metastatic SKCM patients is only $4.6 \%$. Therefore, there is an urgent need to identify potential prognosis-related genes for SKCM and provide more powerful gene markers for the diagnosis of metastatic prognosis in SKCM patients.

In this study, we performed a comprehensive bioinformatics analysis based on the gene expression profile datasets (TCGA and GSE65904 databases) of SKCM patients. By classifying and comparing the gene expression data in the training dataset, we found 876 DEGs, of which 353 were downregulated and 523 were upregulated. With univariate and multivariate Cox regression analysis in the survival package of R3.4.1, we screened 61 independent prognostic DEGs, and then using LR and cluster analysis, we successfully screened 11 feature DEGs: ZNF750, NLRP6, TGM3, KRTDAP, CAMSAP3, KRT6C, CALML5, $S P R R 2 E$, CD3G, RTP5, and FAM83C. From among these 11 feature DEGs, we screened feature DEGs that significantly increased or decreased between cluster 1 and cluster 2 in the training dataset with $P<0.001$. To understand the biological functions of the screened DEGs, we used the STRING database to search for interactions between the products of screened DEGs and constructed a PPI network. Then, we used the DAVID online tool perform GO function and KEGG signaling pathway enrichment analysis of genes on the PPI network. Of the screened 9 KEGG signaling pathways, one is worth pointing out: "hsa04916: Melanogenesis." There are two genes involved in this pathway, CALML3 and CALML5, of which $C A L M L 5$ is one of the important feature DEGs.

CALML5 encodes the CALML5 protein with 146 amino acids. Unlike the generally expressed calmodulin, CALML5 expression is limited to the epidermis and other layered epithelial tissues, and it is highly expressed in the differentiated epidermis [23]. In this study, we found that CALML5 is involved in epidermal development, the calcium signaling pathway, and vascular smooth muscle contraction. Previous studies have reported 


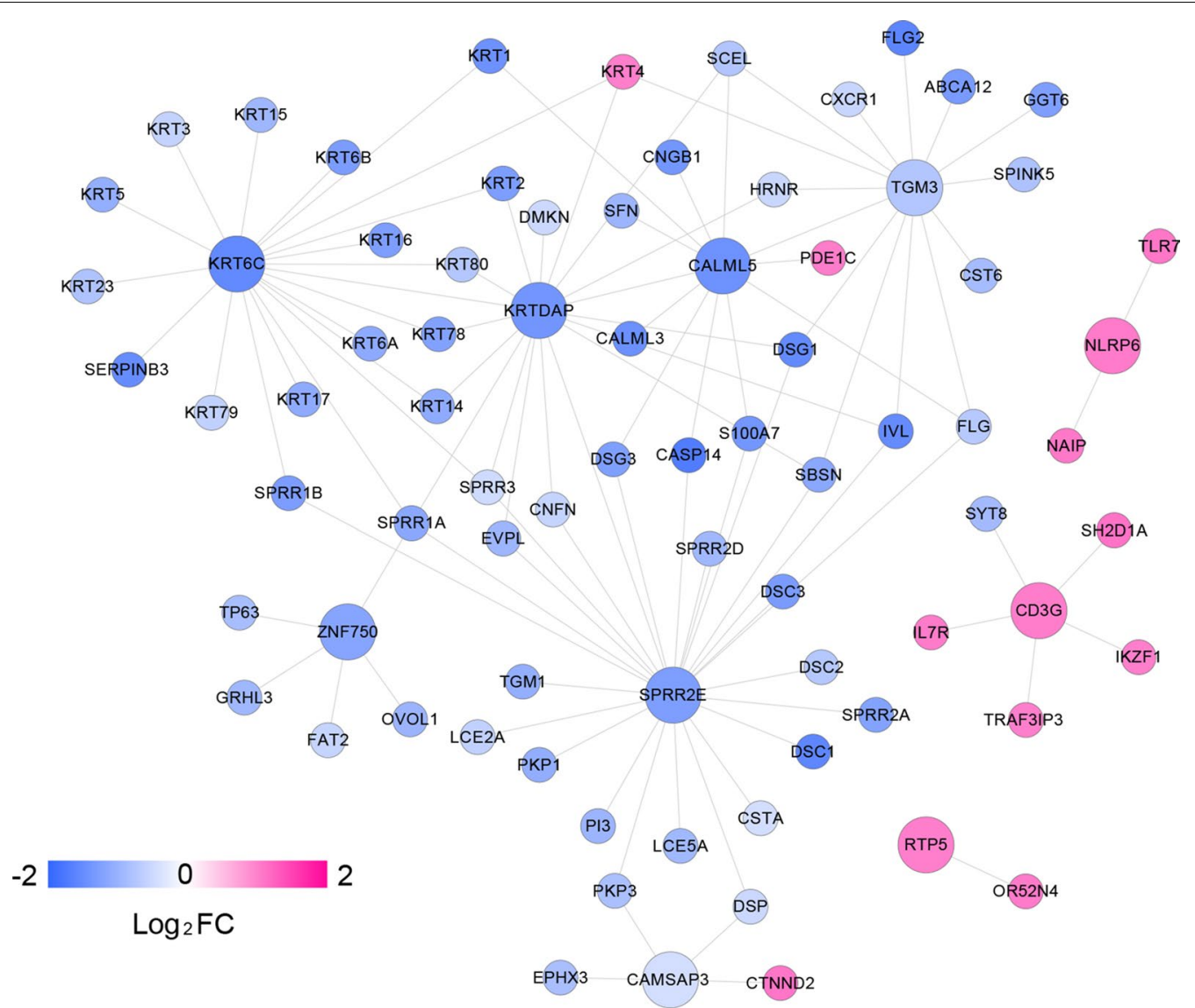

Fig. 5 PPI network diagrams related to the feature DEGs. The change in color from blue to red indicates the change in expression from low to high in the comparison of metastatic vs. nonmetastatic SKCM, and the larger nodes indicate feature DEGs. PPI, protein-protein interaction; DEGs, differentially expressed genes; SKCM, skin cutaneous melanoma

that CALML5 is involved in terminal differentiation of keratinocytes and encodes a calcium-binding protein expressed in the epidermis [24]. In addition, CALML5, as a calmodulin-like protein, participates in not only epidermal differentiation but also intracellular signal transduction. Therefore, CALML5 plays an important role in cell proliferation, differentiation, apoptosis, and migration [25]. Kurozumi et al. showed that CALML5 is a key gene for lymphatic vascular infiltration in early breast cancer and has potential prognostic value [26]. In addition, CALML5 is involved in the "hsa04916: Melanogenesis" pathway. Mac et al. [27] showed that calmodulin-like protein can the SKCM cell growth. In addition, Ke et al. [28] found that CALML5 may be considered a novel biomarker for lung adenocarcinoma diagnosis, which is useful for predicting the risk of lung adenocarcinoma. Misawa et al. [29] found that CALML5 has a high predictive ability as an emerging biomarker for a validation set, capable of discriminating between the plasma of patients and healthy individuals. However, a few studies have reported an association between CALML5 and SKCM, although the mechanism is unclear. Our results will provide a novel view for the prognosis of SKCM.

In this study, we collected 12 targets of CALML5, including TGM3, PDE1C, and CASP14. Smirnov et al. [30] showed that TGM3 is absent in melanocytes as well as SKCM samples and that the expression pattern of TGM3 renders it a potential specific marker for basal cell carcinoma diagnosis. Hu et al. [31] reported that TGM3 controls multiple oncogenic pathways in hepatocellular carcinoma (HCC), contributing to increased cell proliferation and epithelial-mesenchymal transition (EMT). TGM3 also potentially enhances HCC metastasis. TGM3 may serve as a novel therapeutic target in HCC. Wu et al. [32] found that PDE1C is associated with SKCM development. Shimizu et al. found that PDE1C messenger RNA is expressed and may play an important role in human malignant SKCM melanoma-associated antigen cells. Chen et al. [33] reported that CASP14 might be a potential biomarker for gastric cancer diagnosis and an independent prognostic factor of gastric cancer. These results indicate that TGM3 may be an important target 
Table 5 GO biological processes and KEGG signaling pathways in which genes are significantly related in the interaction network

\begin{tabular}{|c|c|c|c|c|}
\hline Category & Term & Count & Gene & $P$ value \\
\hline \multirow[t]{21}{*}{ Biology Process } & GO:0,031,424 keratinization & 20 & TGM3, HRNR & $3.18 \mathrm{E}-35$ \\
\hline & GO:0,008,544 epidermis development & 22 & CALML5, ZNF750 & $9.46 \mathrm{E}-34$ \\
\hline & GO:0,030,216 keratinocyte differentiation & 19 & TGM3, TP63 & $1.04 \mathrm{E}-28$ \\
\hline & GO:0,018,149 peptide cross-linking & 14 & LCE5A, EVPL & $8.98 \mathrm{E}-22$ \\
\hline & GO:0,061,436 establishment of skin barrier & 9 & HRNR, FLG & $2.25 \mathrm{E}-16$ \\
\hline & GO:0,045,104 intermediate filament cytoskeleton organization & 4 & KRT6C, KRT3 & $2.92 \mathrm{E}-06$ \\
\hline & GO:0,043,163 cell envelope organization & 3 & HRNR, TGM3 & 4.98E-06 \\
\hline & GO:0,016,337 single organismal cell-cell adhesion & 6 & PKP1, DSG1 & 5.90E-06 \\
\hline & GO:0,042,060 wound healing & 5 & NLRP6, KRT6A & $3.26 \mathrm{E}-05$ \\
\hline & GO:0,045,109 intermediate filament organization & 3 & KRT17, KRT2 & $1.92 \mathrm{E}-04$ \\
\hline & GO:0,030,855 epithelial cell differentiation & 4 & KRT14, KRT3 & 2.99E-04 \\
\hline & GO:0,007,010 cytoskeleton organization & 5 & KRT6B, KRT5 & 4.34E-04 \\
\hline & GO:0,031,069 hair follicle morphogenesis & 3 & KRT17, TGM3 & 5.47E-04 \\
\hline & GO:0,010,482 regulation of epidermal cell division & 2 & TP63, SFN & $1.23 \mathrm{E}-03$ \\
\hline & GO:0,045, $110 \sim$ intermediate filament bundle assembly & 2 & PKP1, KRT14 & 1.23E-03 \\
\hline & GO:0,051,546 keratinocyte migration & 2 & KRT16, KRT2 & 2.04E-03 \\
\hline & GO:0,045,087 innate immune response & 6 & NLRP6,SH2D1A & $3.18 \mathrm{E}-03$ \\
\hline & GO:0,010,838 positive regulation of keratinocyte proliferation & 2 & TGM1, TP63 & $3.64 \mathrm{E}-03$ \\
\hline & GO:0,003,334 keratinocyte development & 2 & KRT2, SFN & 4.04E-03 \\
\hline & GO:0,031,581 hemidesmosome assembly & 2 & KRT, KRT14 & 4.82E-03 \\
\hline & GO:0,043,616 keratinocyte proliferation & 2 & TP63, KRT2 & 4.82E-03 \\
\hline \multirow[t]{9}{*}{ KEGG Pathway } & hsa04020:Calcium signaling pathway & 3 & CALML3, CALML5 & $9.31 \mathrm{E}-03$ \\
\hline & hsa04070:Phosphatidylinositol signaling system & 2 & CALML3, CALML5 & $1.98 \mathrm{E}-02$ \\
\hline & hsa04640:Hematopoietic cell lineage & 2 & CD3G, IL7R & $2.26 \mathrm{E}-02$ \\
\hline & hsa04912:GnRH signaling pathway & 2 & CALML3, CALML5 & $2.53 \mathrm{E}-02$ \\
\hline & hsa04916:Melanogenesis & 2 & CALML3, CALML5 & $2.56 \mathrm{E}-02$ \\
\hline & hsa04114:Oocyte meiosis & 2 & CALML3, CALML5 & 2.80E-02 \\
\hline & hsa04270:Vascular smooth muscle contraction & 2 & CALML3, CALML5 & $2.84 \mathrm{E}-02$ \\
\hline & hsa04722:Neurotrophin signaling pathway & 2 & CALML3, CALML5 & 3.10E-02 \\
\hline & hsa04910:Insulin signaling pathway & 2 & CALML3, CALML5 & $3.32 \mathrm{E}-02$ \\
\hline
\end{tabular}

gene for SKCM treatment. Thus, CALML5 may play an important role in SKCM metastasis and promote SKCM occurrence and development, so CALML5 may become a potential target for the treatment of SKCM metastasis in the future.

Another calmodulin-like protein, $C A L M L 3$, is a tumor suppressor gene, which significantly inhibits liver cancer growth and lung metastasis. CALML3 is a gene with "early warning" value for liver cancer and lung metastasis, and it is expected to become a new marker for early diagnosis of lung metastasis of liver cancer and a new target for inhibiting liver cancer growth and lung metastasis [34]. These findings show that CALML3 may be closely related to SKCM metastasis and prognosis, and that it has certain clinical significance for the prediction of SKCM and its prognosis.
Although we identified 11 feature DEGs related to SKCM metastasis, the detailed mechanisms have not yet been explored. For example, a further accurate classification with a large sample size and clinical information needs to identify SKCM metastatic and nonmetastatic patients. In addition, whether the 11 feature DEGs are involved in several molecular pathways, such as hsa04916: Melanogenesis, needs to be investigated. Corresponding experimental research is also needed to verify feature gene functions.

\section{Conclusion}

We identified 876 DEGs (353 downregulated and 523 upregulated) in the TCGA training dataset. In addition, 11 important prognostic-related feature DEGs, such as ZNF750, NLRP6, TGM3, CALML5, CD3G, and RTP5, may play an important role in SKCM metastasis. These 
DEGs are involved in 9 KEGG signaling pathways, such as the "hsa04916: Melanogenesis" pathway. Of the 11 feature DEGs, CALML5 is involved in the "hsa04916: Melanogenesis" pathway, 12 targets of which were collected, such as TGM3, PDE1C, and CASP14. This study provides new ideas for exploring the molecular mechanism underlying SKCM metastasis and finding new diagnostic prognostic markers.

\section{Abbreviations}

DEGs: Differentially expressed genes; GO: Gene Ontology; KEGG: Kyoto Encyclopedia of Genes and Genomes; PPI: Protein-protein interaction; SKCM: Skin cutaneous melanoma; TCGA: The Cancer Genome Atlas.

\section{Acknowledgements}

Not applicable.

\section{Authors' contributions}

YW and GJ were responsible for the conception and design of the research, and drafting the manuscript. ZS performed the acquisition, analysis, and interpretation of the data. ZX and YT performed the data analysis and interpretation. XW participated in the design of the study and performed the statistical analysis. All authors read and approved the manuscript.

\section{Funding}

None.

\section{Availability of data and materials}

The datasets supporting the conclusions of this article are available in the [GSE65904] and [TCGA] repository, [https://www.ncbi.n/m.nih.gov/geo/query/ acc.cgi?acc=GSE65904] and [https://xenabrowser.net/datapages/?cohort= GDC\%20TCGA\%20Melanoma\%20(SKCM)\&removeHub=https\%3A\%2F\% 2Fxena.treehouse.gi.ucsc.edu\%3A443].

\section{Declarations}

Ethics approval and consent to participate

Not applicable.

\section{Consent for publication}

Not applicable.

\section{Competing interests}

The authors declare that they have no competing interests.

\section{Author details}

${ }_{1}^{1}$ Department of Orthopedics, The Second Clinical Hospital of Jilin University, NO.218, Ziqiang Street, Nanguan District, Changchun 130000, Jilin, China.

${ }^{2}$ Department of Gastrointestinal and Colorectal Surgery, The Third Hospital of Jilin University, No.126, Xiantai Street, Changchun 130033, Jilin, China.

${ }^{3}$ Department of Brest Surgery, The Second Clinical Hospital of Jilin University, NO.218, Ziqiang Street, Nanguan District, Changchun 130000, Jilin, China.

Received: 30 Auqust 2020 Accepted: 25 February 2021

Published online: 06 April 2021

\section{References}

1. Shah DJ, Dronca RS. Latest advances in chemotherapeutic, targeted, and immune approaches in the treatment of metastatic melanoma. Mayo Clin Proc. 2014;89(4):504-19.

2. Leonardi GC, Falzone L, Salemi R, Zanghì A, Spandidos DA, McCubrey JA, et al. Cutaneous melanoma: from pathogenesis to therapy (Review). Int J Oncol. 2018;52(4):1071-80.
3. Xiong TF, Pan FQ, Li D. Expression and clinical significance of S100 family genes in patients with melanoma. Melanoma Res. 2019;29(1):23-9.

4. Wagner NB, Forschner A, Leiter U, Garbe C, Eigentler TK. S100B and $\mathrm{LDH}$ as early prognostic markers for response and overall survival in melanoma patients treated with anti-PD-1 or combined anti-PD-1 plus anti-CTLA-4 antibodies. Br J Cancer. 2018;1 19(3):339-46.

5. Da Forno PD, Pringle JH, Hutchinson P, Osborn J, Huang Q, Potter L, et al. WNT5A expression increases during melanoma progression and correlates with outcome. Clin Cancer Res. 2008;14(18):5825-32.

6. Bosserhoff AK. Melanoma inhibitory activity (MIA): an important molecule in melanoma development and progression. Pigment Cell Res. 2005;18(6):411-6.

7. Ci C, Tang B, Lyu D, Liu W, Qiang D, Ji X, et al. Overexpression of CDCA8 promotes the malignant progression of cutaneous melanoma and leads to poor prognosis. Int J Mol Med. 2019;43(1):404-12.

8. Yang L, Xu Y, Yan Y, Luo P, Chen S, Zheng B, et al. Common nevus and skin cutaneous melanoma: prognostic genes identified by gene co-expression network analysis. Genes (Basel). 2019;10(10):747.

9. Bisaso KR, Karungi SA, Kiragga A, Mukonzo JK, Castelnuovo B. A comparative study of logistic regression based machine learning techniques for prediction of early virological suppression in antiretroviral initiating HIV patients. BMC Med Inform Decis Mak. 2018;18(1):77.

10. Zejnullahu VA, Ukella-Lleshi D, Zejnullahu VA, Miftari E, Govori V. Prevalence of postpartum depression at the clinic for obstetrics and gynecology in Kosovo teaching hospital: Demographic, obstetric and psychosocial risk factors. Eur J Obstet Gynecol Reprod Biol. 2021;256:215-20.

11. Sufriyana H, Husnayain A, Chen YL, Kuo CY, Singh O, Yeh TY, et al. Comparison of multivariable logistic regression and other machine learning algorithms for prognostic prediction studies in pregnancy care: systematic review and meta-analysis. JMIR Med Inform. 2020;8(11):e16503.

12. Sokou R, Piovani D, Konstantinidi A, Tsantes AG, Parastatidou S, Lampridou $\mathrm{M}$, et al. A risk score for predicting the incidence of hemorrhage in critically ill neonates: development and validation study. Thromb Haemost. 2021;121:131-9.

13. Wang $P$, Wang $Y$, Hang B, Zou X, Mao JH. A novel gene expression-based prognostic scoring system to predict survival in gastric cancer. Oncotarget. 2016;7(34):55343-51.

14. Lee JW, Lee JB, Park M, Song SH. An extensive comparison of recent classification tools applied to microarray data. Comput Stat Data Anal. 2005;48(4):869-85.

15. Zhu J, Hastie T. Classification of gene microarrays by penalized logistic regression. Biostatistics (Oxford, England). 2004;5(3):427-43.

16. Wang $L$, Cao $C, M a ~ Q$, Zeng $Q$, Wang $H$, Cheng Z, et al. RNA-seq analyses of multiple meristems of soybean: novel and alternative transcripts, evolutionary and functional implications. BMC Plant Biol. 2014;14:169.

17. Szklarczyk D, Gable AL, Lyon D, Junge A, Wyder S, Huerta-Cepas J, et al. STRING v11: protein-protein association networks with increased coverage, supporting functional discovery in genome-wide experimental datasets. Nucleic Acids Res. 2019;47(D1):D607-13.

18. Shannon P, Markiel A, Ozier O, Baliga NS, Wang JT, Ramage D, et al. Cytoscape: a software environment for integrated models of biomolecular interaction networks. Genome Res. 2003;13(11):2498-504.

19. da Huang W, Sherman BT, Lempicki RA. Systematic and integrative analysis of large gene lists using DAVID bioinformatics resources. Nat Protoc. 2009:4(1):44-57.

20. da Huang W, Sherman BT, Lempicki RA. Bioinformatics enrichment tools: paths toward the comprehensive functional analysis of large gene lists. Nucleic Acids Res. 2009;37(1):1-13.

21. Zhang X, Ding C, Tian H, Dong X, Meng X, Zhu W, et al. ZNF23 suppresses cutaneous melanoma cell malignancy via mitochondria-dependent pathway. Cellular Physiol Biochem. 2017:43(1):147-57.

22. Cohen I, Birnbaum RY, Leibson K, Taube R, Sivan S, Birk OS. ZNF750 is expressed in differentiated keratinocytes and regulates epidermal late differentiation genes. PLoS ONE. 2012;7(8):e42628.

23. Babini E, Bertini I, Capozzi F, Chirivino E, Luchinat C. A structural and dynamic characterization of the EF-hand protein CLSP. Structure. 2006;14(6):1029-38.

24. Donovan M, Ambach A, Thomas-Collignon A, Prado C, Bernard D, Jammayrac $\mathrm{O}$, et al. Calmodulin-like skin protein level increases in the differentiated epidermal layers in atopic dermatitis. Exp Dermatol. 2013;22(12):836-7. 
25. Villalobo A, González-Muñoz M, Berchtold MW. Proteins with calmodulinlike domains: structures and functional roles. Cellular and molecular life sciences: CMLS. 2019;76(12):2299-328.

26. Kurozumi S, Joseph C, Sonbul S, Alsaeed S, Kariri Y, Aljohani A, et al. A key genomic subtype associated with lymphovascular invasion in invasive breast cancer. Br J Cancer. 2019;120(12):1129-36.

27. Mac Neil S, Walker SW, Senior HJ, Bleehen SS, Tomlinson S. Effects of extracellular calmodulin and calmodulin antagonists on B16 melanoma cell growth. J Invest Dermatol. 1984:83(1):15-9.

28. Ke H, Wu Y, Wang R, Wu X. Creation of a prognostic risk prediction model for lung adenocarcinoma based on gene expression, methylation, and clinical characteristics. Med Sci Monit. 2020;26:e925833.

29. Misawa K, Imai A, Matsui H, Kanai A, Misawa Y, Mochizuki D, et al. Identification of novel methylation markers in HPV-associated oropharyngeal cancer: genome-wide discovery, tissue verification and validation testing in ctDNA. Oncogene. 2020;39(24):4741-55.

30. Smirnov A, Anemona L, Montanaro M, Mauriello A, Annicchiarico-Petruzzelli M, Campione E, et al. Transglutaminase 3 is expressed in basal cell carcinoma of the skin. Eur J Dermatol. 2019;29(5):477-83.
31. Hu JW, Yang ZF, Li J, Hu B, Luo CB, Zhu K, et al. TGM3 promotes epithelialmesenchymal transition and hepatocellular carcinogenesis and predicts poor prognosis for patients after curative resection. Dig Liver Dis. 2020;52(6):668-76.

32. Wu W, Liu H, Song F, Chen LS, Kraft P, Wei Q, et al. Associations between smoking behavior-related alleles and the risk of melanoma. Oncotarget. 2016;7(30):47366-75.

33. Chen $\mathrm{Q}$, Huang $X$, Dong $X$, Wu J, Teng F, Xu H. Long non-coding RNA ERICH3-AS1 is an unfavorable prognostic factor for gastric cancer. PeerJ. 2020;8:e8050

34. Yang B, Li M, Tang W, Liu W, Zhang S, Chen L, et al. Dynamic network biomarker indicates pulmonary metastasis at the tipping point of hepatocellular carcinoma. Nat Commun. 2018;9(1):678.

\section{Publisher's Note}

Springer Nature remains neutral with regard to jurisdictional claims in published maps and institutional affiliations.
Ready to submit your research? Choose BMC and benefit from:

- fast, convenient online submission

- thorough peer review by experienced researchers in your field

- rapid publication on acceptance

- support for research data, including large and complex data types

- gold Open Access which fosters wider collaboration and increased citations

- maximum visibility for your research: over 100M website views per year

At BMC, research is always in progress.

Learn more biomedcentral.com/submissions 